UCB-PTH-02/55

LBNL-51782

FERMILAB-Conf-02/322-T

\title{
Grand Unification in Higher Dimensions
}

\author{
Lawrence J. Hall ${ }^{a, b}$ and Yasunori Nomura ${ }^{c}$ \\ ${ }^{a}$ Department of Physics, University of California, Berkeley, CA 94720 \\ ${ }^{b}$ Theoretical Physics Group, Lawrence Berkeley National Laboratory, Berkeley, CA 94720 \\ ${ }^{c}$ Theoretical Physics Department, Fermi National Accelerator Laboratory, Batavia, IL 60510
}

\begin{abstract}
We have recently proposed an alternative picture for the physics at the scale of gauge coupling unification, where the unified symmetry is realized in higher dimensions but is broken locally by a symmetry breaking defect. Gauge coupling unification, the quantum numbers of quarks and leptons and the longevity of the proton arise as phenomena of the symmetrical bulk, while the lightness of the Higgs doublets and the masses of the light quarks and leptons probe the symmetry breaking defect. Moreover, the framework is extremely predictive if the effective higher dimensional theory is valid over a large energy interval up to the scale of strong coupling. Precise agreement with experiments is obtained in the simplest theory - $S U(5)$ in five dimensions with two Higgs multiplets propagating in the bulk. The weak mixing angle is predicted to be $\sin ^{2} \theta_{w}=0.2313 \pm 0.0004$, which fits the data with extraordinary accuracy. The compactification scale and the strong coupling scale are determined to be $M_{c} \simeq 5 \times 10^{14} \mathrm{GeV}$ and $M_{s} \simeq 1 \times 10^{17} \mathrm{GeV}$, respectively. Proton decay with a lifetime of order $10^{34}$ years is expected with a variety of final states such as $e^{+} \pi^{0}$, and several aspects of flavor, including large neutrino mixing angles, are understood by the geometrical locations of the matter fields. When combined with a particular supersymmetry breaking mechanism, the theory predicts large lepton flavor violating $\mu \rightarrow e$ and $\tau \rightarrow \mu$ transitions, with all superpartner masses determined by only two free parameters. The predicted value of the bottom quark mass from Yukawa unification agrees well with the data. This paper is mainly a review of the work presented in hep-ph/0103125, hep-ph/0111068 and hep-ph/0205067 [1, 2, 3].
\end{abstract}




\section{Introduction: Features of 4D Grand Unification}

While the manifestations of the strong, weak and electromagnetic forces are very different in nature, these three interactions all follow from local gauge symmetry, suggesting that they may be low energy remnants of a single large gauge symmetry at high energies. Such a grand unified interaction would be described by a single gauge coupling, leading to a correlation among the strengths of the three forces measured at lower energies. Remarkably, this prediction from gauge coupling unification is highly successful, if nature is supersymmetric above the scale of the weak interactions. It implies that the unification of the strong, and electroweak interactions occurs at a mass scale of order $10^{16} \mathrm{GeV}$, but what is the nature of the physical theory underlying this unification and how can it be experimentally tested?

The conventional answer of four dimensional (4D) grand unification [4] shows a remarkable dichotomy: parts of the standard model cry out for 4D unification into a gauge group such as $S U(5)$, while other parts abhor such a unification. For example the quantum numbers of a generation of quarks and leptons fit beautifully into unified representations, providing an elegant understanding of the various gauge quantum numbers $[5,4,6]$, while the Higgs doublet resists unification. The $S U(5)$ partner of the Higgs doublet, $H_{3}$, must be heavy to avoid rapid proton decay and also because it would spoil gauge coupling unification. Whilst the simplest picture of supersymmetric grand unification gives us a very significant prediction for the weak mixing angle $[7,8]$, it also leads to a prediction for proton decay from the exchange of the superheavy triplets $H_{3}$ [9], in strong disagreement with data [10]. Finally, the mass ratio of quarks and leptons in the third generation, $m_{b} / m_{\tau}$, shows a simple ratio which follows directly from grand unification [11], while light quark-lepton mass ratios, such as $m_{s} / m_{\mu}$, do not have values that follow simply from unification. Thus the minimal theory does not explain why there is a light Higgs boson, is excluded by proton decay, and introduces flavor conundrums. Of course, this dichotomy does not exclude supersymmetric 4D unification which has been so much discussed for over 20 years; rather, within these theories we are led to invent a series of mechanisms for doublet-triplet splitting, proton decay suppression, and flavor. However, the resulting theories then acquire a certain level of complexity. Can the dichotomy be resolved more elegantly in an alternative framework?

The prediction of the QCD gauge coupling from 4D supersymmetric unification is good but certainly not perfect, as illustrated in Figure 1. The effect of the supersymmetric logarithm is to greatly improve the prediction, but there is an overshoot beyond the experimental value of $\alpha_{s}\left(M_{Z}\right)=0.117 \pm 0.002[12]$ to $\alpha_{s}\left(M_{Z}\right) \simeq 0.130$ [13]. We typically assume that most of this discrepancy comes from the unification scale, and is due to the complications to the theory that we have been forced to add. Since these corrections involve additional free parameters, they cannot be numerically evaluated. In this talk we will argue that there is an alternative picture 


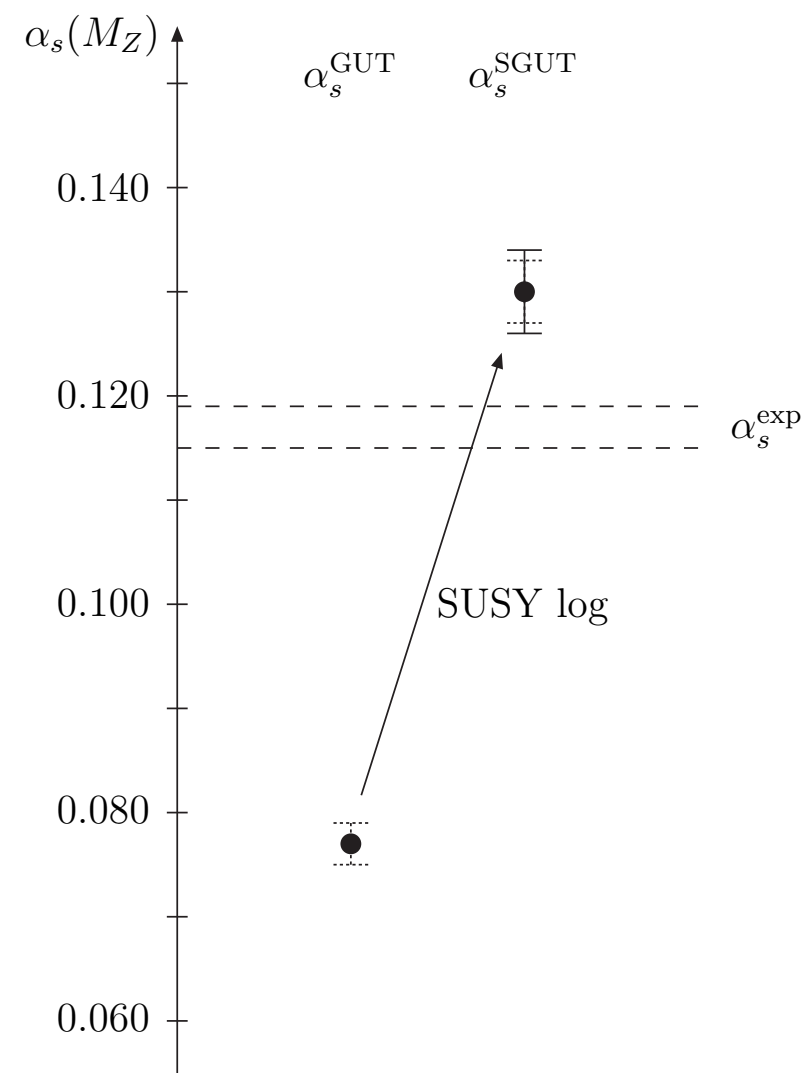

Figure 1: The predictions for $\alpha_{s}\left(M_{Z}\right)$ in non-supersymmetric grand unification, $\alpha_{s}^{\mathrm{GUT}}$, and supersymmetric grand unification, $\alpha_{s}^{\mathrm{SGUT}}$. The solid error bar represents the threshold corrections from the superpartner spectrum. Dotted error bars represent threshold corrections from the unified scale corresponding to a heavy $\mathbf{5}+\overline{\mathbf{5}}$ representation with unit logarithmic mass splitting between doublets and triplets. 
for the physics at the unification scale, and that all aspects of the dichotomy are reconciled in the simplest model. In the next section we introduce the new picture of symmetry breaking defects in a higher dimensional spacetime, and argue that the traditional problems are all elegantly solved. In section 3 we discuss the predictions from gauge coupling and Yukawa coupling unification in the simplest model. The corrections to the QCD gauge coupling from physics at the unified scale do not depend on any extra free parameters, and yield precisely the observed value. The location of matter in the higher dimension is discussed, and predictions for proton decay are given. In section 4 we introduce a new origin for supersymmetry breaking in unified theories. Combining this with the minimal model, predictions are given for the superpartner and Higgs spectrum, for the bottom quark mass from Yukawa unification, and for flavor changing lepton decays. We conclude in section 5.

\section{New Physics for Grand Unification}

Building on the ideas and tools developed by others, over the last year or so we have introduced a new picture for the physics in the energy range of $10^{15}-10^{17} \mathrm{GeV}[1,2,3]$. We describe the new physical picture in this section, and the simplest model for its implementation in the next.

At the $\mathrm{TeV}$ scale we live in a $4 \mathrm{D}$ world, spanned by the coordinates $x$, and the gauge group is $S U(3)_{C} \times S U(2)_{L} \times U(1)_{Y}(3-2-1)$, as illustrated by the sheet on the left-hand side of Figure 2 . At the unification scale we suppose that other dimensions of size $R$ are resolved, described by coordinates $y$, as shown on the right of the figure. It is the mass scale $1 / R$, rather than the expectation value of some field, that characterizes the scale of unification. Particles moving in the $y$ direction can be viewed as particles moving in a box of size $R$ and therefore have momenta $p_{y}$ quantized in units of $1 / R$. To observers in $4 \mathrm{D}$, particles with different $p_{y}$ appear as particles of different mass, so that there is a discrete tower of particles, known as the Kaluza-Klein (KK) tower. A crucial aspect of our physical picture is the structure of the gauge symmetries in the box of the $y$ direction. Interactions in the interior of the box are symmetrical under the full gauge symmetry $G$ of the unified theory, while those on a boundary are only symmetrical under the standard model 3-2-1 gauge symmetries, as shown in Figure 2.

Since our own four dimensions are known, it is convenient to suppress $x$ and display only the extra dimensions $y$, as illustrated in Figure 3 for the case of three extra dimensions. The space of the extra dimensions is known as the bulk, and we will also refer to it as a box. The sizes of the extra dimensions need not be the same, although we imagine they are not extremely different, and, while we have shown a simple box, the bulk may have a more complicated geometry. The crucial point is that the gauge group throughout the volume of this extra-dimensional bulk is the unified group $G$, while that of the standard model appears only on some lower dimensional 


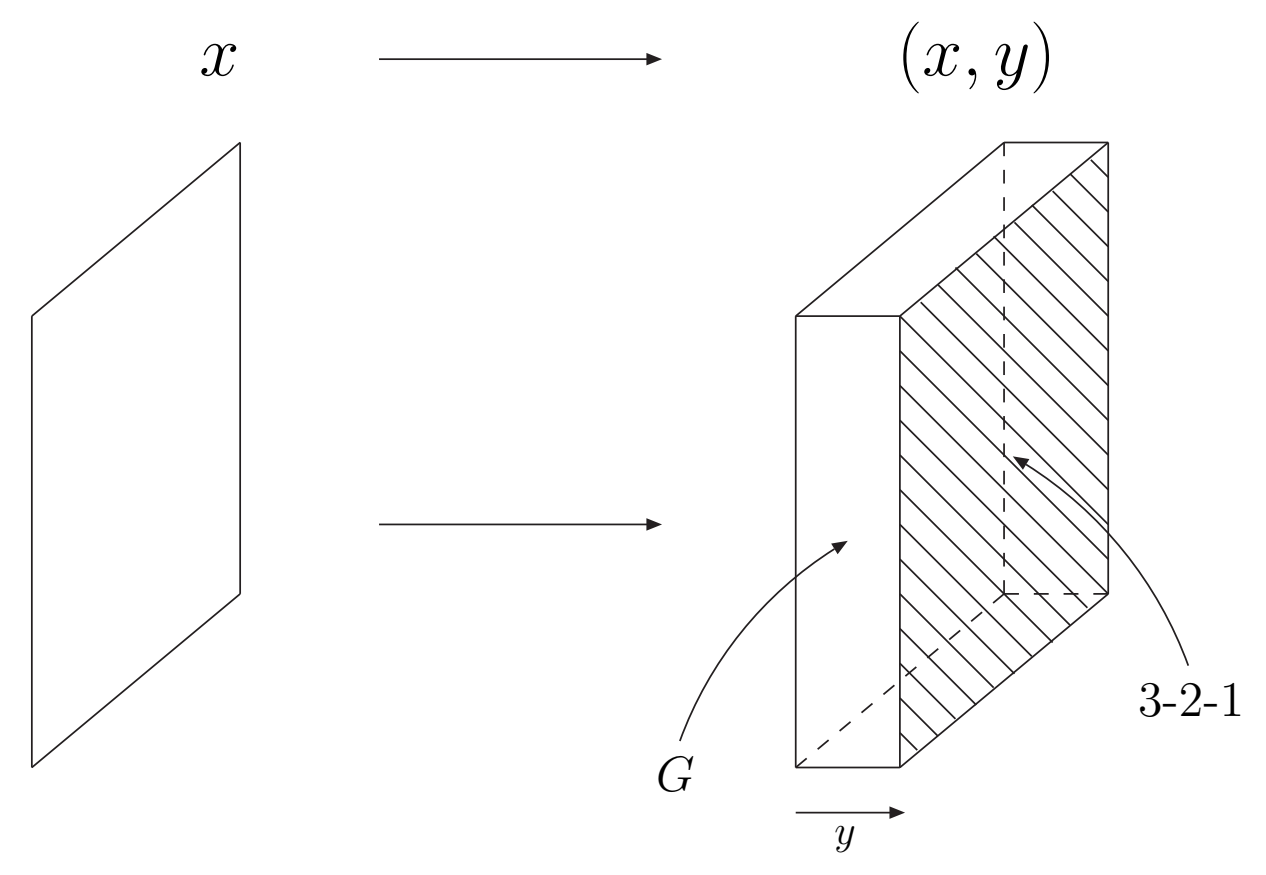

Figure 2: Physics at high energies probes extra dimensions, $y$, that extend over small sizes $R$. The interactions in the volume of this higher dimensional box are constrained by a unified gauge symmetry $G$, but interactions on a boundary may be constrained only by a smaller symmetry, such as 3-2-1, creating a defect of lower dimension. 


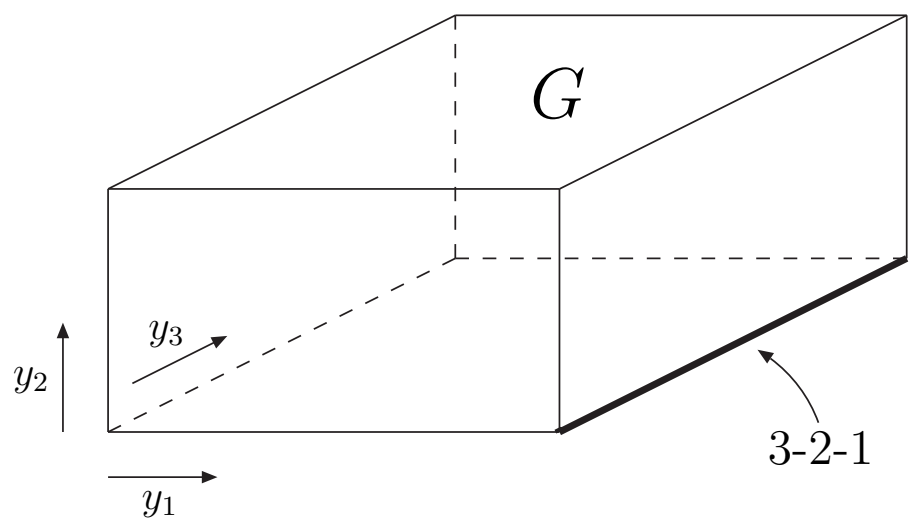

Figure 3: An example of a 3D bulk with a 1D defect.

boundary surface. In Figure 3 we have shown the 3-2-1 surface as having dimension 1, but it could be a 2D surface, or it could be a point at one of the corners of the box. In our picture, most of spacetime feels the full gauge invariance of $G$, while there is a defect on a lower dimensional surface which only feels the 3-2-1 gauge symmetry. The symmetry breaking therefore appears explicitly, as a spatial defect - a complete change of viewpoint compared to 4D grand unification! One might naively guess that such local defects could not lead to the world we see - where 3-2-1 forces are observed to be quite different from each other, and the other interactions in $G$ are incredibly feeble. Figure 3 gives the impression that the breaking of $G$ is minor and perhaps just a small correction. For short distance physics in the bulk this is certainly true - but for long distance physics the boundary effects become all important, as they determine the light states of the theory.

How does this new picture reconcile the dichotomy of 4D unification? The idea is remarkably simple: the aspects which fit unification so well, gauge coupling unification, quark and lepton quantum numbers and $m_{b} / m_{\tau}$, should be phenomena of the regions of the bulk where the physics is unified and should be insensitive to the 3-2-1 defect, while the aspects which abhor unification, such as the light Higgs doublet and $m_{s} / m_{\mu}$, should probe the 3-2-1 defect in a nontrivial way. It will turn out that the suppression of proton decay results from the enlargement of the spacetime symmetry of the bulk.

What is the origin of the 3-2-1 defect? In particular what determines its location and why is it on a boundary of the bulk rather than somewhere in the interior? We will answer this in the context of the effective higher dimensional field theory. We do not explain the origin of the extra dimensions nor their size, but, given this enlarged spacetime, we write the most general theory subject to a set of symmetries. The new ingredient here, compared with familiar 4D theories, 


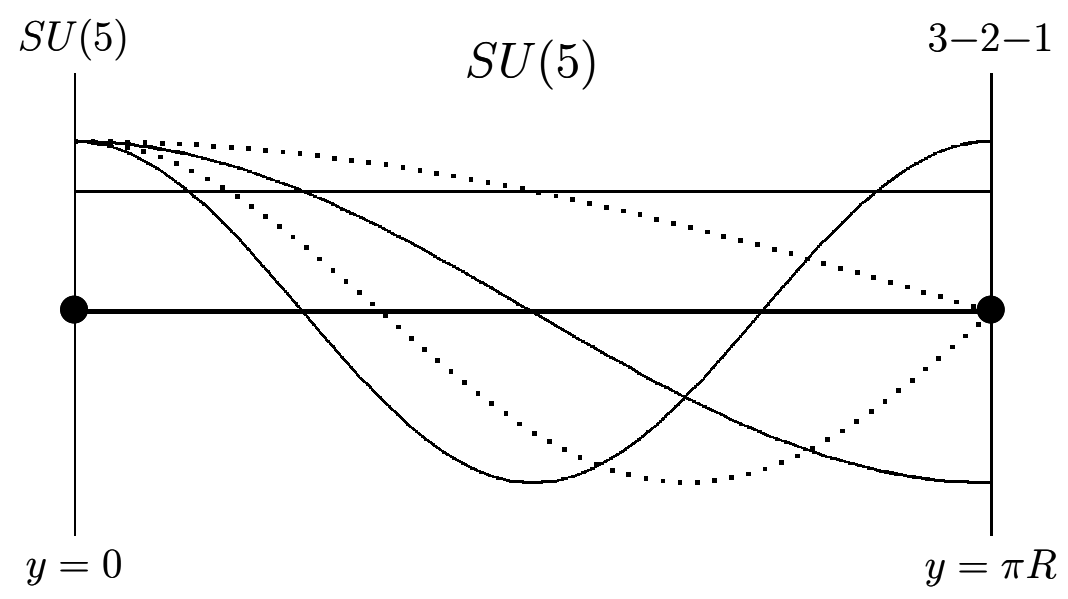

Figure 4: In the fifth dimension, space is a line segment with boundaries at $y=0$ and at $y=\pi R$. Solid and dotted lines represent the profiles of gauge transformation parameters $\xi_{321}$ and $\xi_{X}$, respectively. Because $\xi_{X}(y=\pi R)=0$, a point defect occurs in the symmetry at the $y=\pi R$ boundary, explicitly breaking $S U(5)$ to $S U(3)_{C} \times S U(2)_{L} \times U(1)_{Y}$.

is that because our spacetime has boundaries we must specify boundary conditions to define the theory. The boundary conditions are chosen not to spoil the consistency of the theory, and, within the effective field theory description, the 3-2-1 defect originates from these boundary conditions. Specifically, in 4D theories we take the gauge parameters for transformation $a$ to be arbitrary functions of spacetime, $\xi_{a}(x)$, but with a finite bulk we must specify boundary conditions for these parameters. In particular, at some boundary $y=y_{b}$ we can specify different conditions on the 3-2-1 gauge parameters, $\xi_{321}\left(y_{b}\right)$, and the remaining gauge parameters of $G$, $\xi_{X}\left(y_{b}\right)$, inducing the $G$-breaking defect on this boundary. For example, if $\xi_{X}\left(y_{b}\right)=0$ and $\xi_{321}\left(y_{b}\right) \neq 0$ then the unified gauge bosons $X$ do not have interactions on this boundary, while the 3-2-1 gauge bosons of the standard model do. This is illustrated for the case $G=S U(5)$ in a $1 \mathrm{D}$ bulk in Figure 4. In this example the fields and interactions at the $y=\pi R$ boundary need only respect 3-2-1 gauge symmetry - they explicitly break the $S U(5)$ symmetry [1].

This is clearly a radical departure from the familiar Higgs mechanism for spontaneously breaking gauge symmetries. There is no Higgs field - in the effective field theory the phenomena are geometrical rather than dynamical. In cosmology, as the temperature $T$ of the universe cools through $1 / R$, there is no phase transition; rather the symmetry breaking effects gradually grow in importance. At $T \gg 1 / R$ they are important only very close to the defect, and irrelevant everywhere else. At lower temperatures they become ever more dominant, and the symmetries of the bulk cannot be resolved. Remarkably, this explicit breaking of gauge symmetry does not destroy calculability of the theory. The unitarity behavior of the theory is 

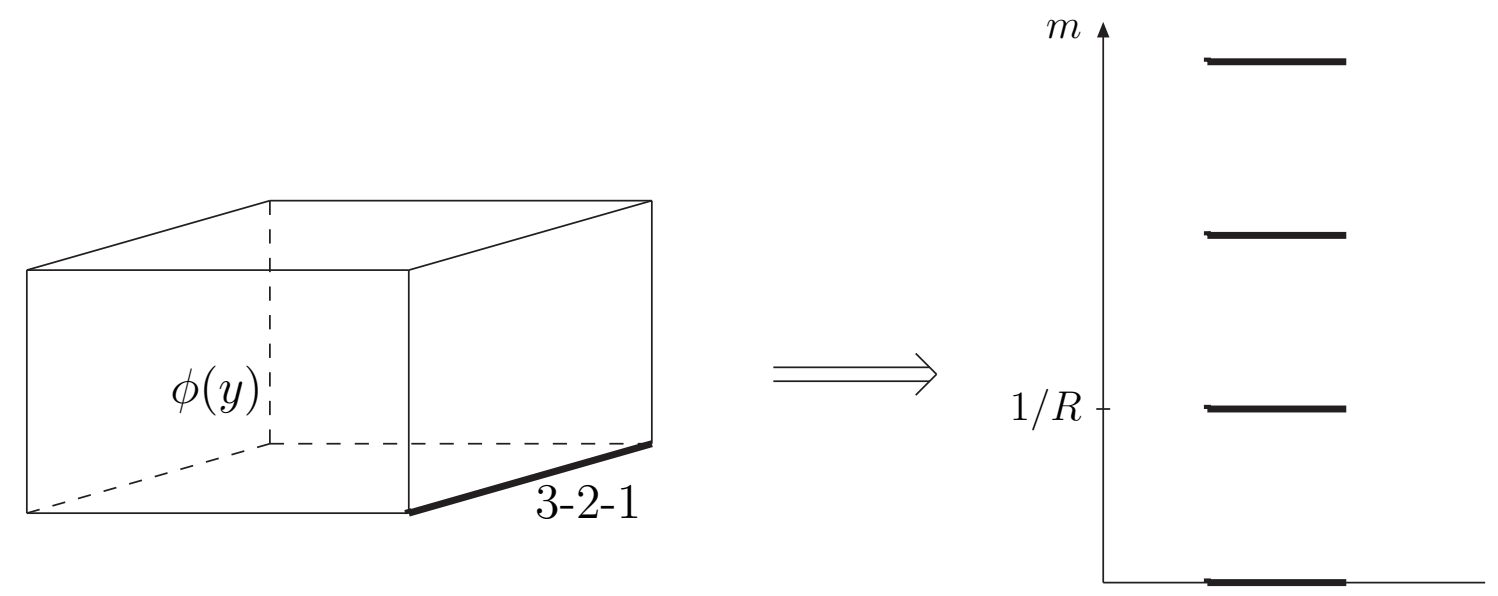

Figure 5: A bulk, having boundary conditions leading to a defect, can be viewed as a "machine" for creating a mass spectrum of $4 \mathrm{D}$ particles, known as a KK tower.

no worse than when the boundary conditions preserve $S U(5)$ [14].

What happens when fields $\phi(x, y)$ propagate in such a higher dimensional spacetime with a gauge symmetry defect induced by non-trivial boundary conditions? The geometry of the box together with the boundary conditions determine the allowed normal modes. This is just the field theory analogue of quantizing a particle in a box, but now the allowed $p_{y}^{2}$ correspond to the allowed $m^{2}$ for a $4 \mathrm{D}$ observer. Thus the box and boundary conditions can be viewed as a machine for creating a KK tower of massive states, as illustrated in Figure 5.

The typical spacing or discreteness, $1 / R$, for the masses of the KK tower is determined by the size of the box, while the gauge quantum numbers at each level is determined by the boundary conditions. The important point is that modes having different 3-2-1 quantum numbers $q_{1}, q_{2}, \cdots$, have different spectra, because of the $G$-breaking boundary conditions. At high energies the discreteness is not so important and the local symmetry $G$ of the bulk is restored, but at low energies the discreteness is crucial and the gauge quantum numbers of the lowest lying states are all important. At very low energies, only the zero mass modes can be excited, and hence a crucial question is how the boundary conditions determine the quantum numbers of these "zero modes". We can turn this around: at the scale of unification we want only the particles of the standard model and their superpartners to be zero modes, so that running the machine backwards we can find out what geometries and boundary conditions are of interest for nature. The remarkable thing is that we can start with a pure gauge theory in the box - there are no mass terms or spontaneous symmetry breakings — and the 4D mass terms of the KK tower arise from the kinetic energies in the extra dimensions. What are the 
consequences of this new viewpoint for gauge coupling unification, the lightness of the Higgs doublet, proton decay, and quark-lepton mass ratios? We will not be surprised to see large changes from the standard picture.

\subsection{Gauge coupling unification}

Since the unified symmetry $G$ is explicitly broken by boundary conditions, it is not obvious that gauge coupling unification is preserved. In fact, gauge coupling unification is generically destroyed due to the presence of local $G$ breaking on the $y=y_{b}$ boundary. To see this, consider the effective field theory above $1 / R$. Since the higher dimensional gauge theory is non-renormalizable, this effective theory must be cut off at some scale $M_{s}$, where the theory is embedded into a more fundamental theory such as string theory. At the scale $M_{s}$, the most general effective action for the gauge kinetic terms is

$$
S=\int d x d y\left[\frac{1}{g_{5}^{2}} F^{2}+\delta\left(y-y_{b}\right) \frac{1}{\tilde{g}_{a}^{2}} F_{a}^{2}\right],
$$

where the first term arises from the interior of the box and is $G$ invariant, while the second term represents non-unified kinetic operators located on the $y=y_{b}$ boundary ( $F$ is the field strength, and $a=1,2,3$ represents the standard model gauge groups). This form is ensured by the $y$-dependent gauge symmetry of our effective theory, regardless of the unknown ultraviolet physics above $M_{s}$. The standard model gauge couplings in the equivalent 4D theory, $g_{a}$, are then obtained by integrating over the extra dimensions:

$$
\frac{1}{g_{a}^{2}}=\frac{R^{d}}{g_{5}^{2}}+\frac{R^{d^{\prime}}}{\tilde{g}_{a}^{2}}
$$

for $d$ extra dimensions of size $R$ and defects of dimension $d^{\prime}<d$. This shows that $g_{a}$ depend on the coefficients of the localized kinetic operators, $\tilde{g}_{a}$, and are not universal at the scale $M_{s}$ in general there is no gauge coupling unification! However, if the extra dimensions have a large size, we find that the $R^{d}$ factor dominates over $R^{d^{\prime}}$, ensuring the unified contribution dominates over the 3-2-1 defect contribution, and gauge coupling unification is recovered [1].

The energy dependence of the gauge couplings in our scheme is shown in Figure 6 . The estimate for the compactification scale, $M_{c} \equiv 1 / R \approx 10^{15} \mathrm{GeV}$, and for the fundamental scale, $M_{s} \approx 10^{17} \mathrm{GeV}$, are for the particular 5D theory with $G=S U(5)$ discussed in the next section, but the general behavior of the running of these couplings is generic to our framework. The couplings do not unify at the compactification scale; rather they continue to evolve even above the compactification scale where the physics is higher dimensional. The higher dimensional behavior of the theory is apparent because of the rapid growth of the interaction strength with energy. Above $M_{c}$ the couplings continue to approach each other because of the $G$-violating 


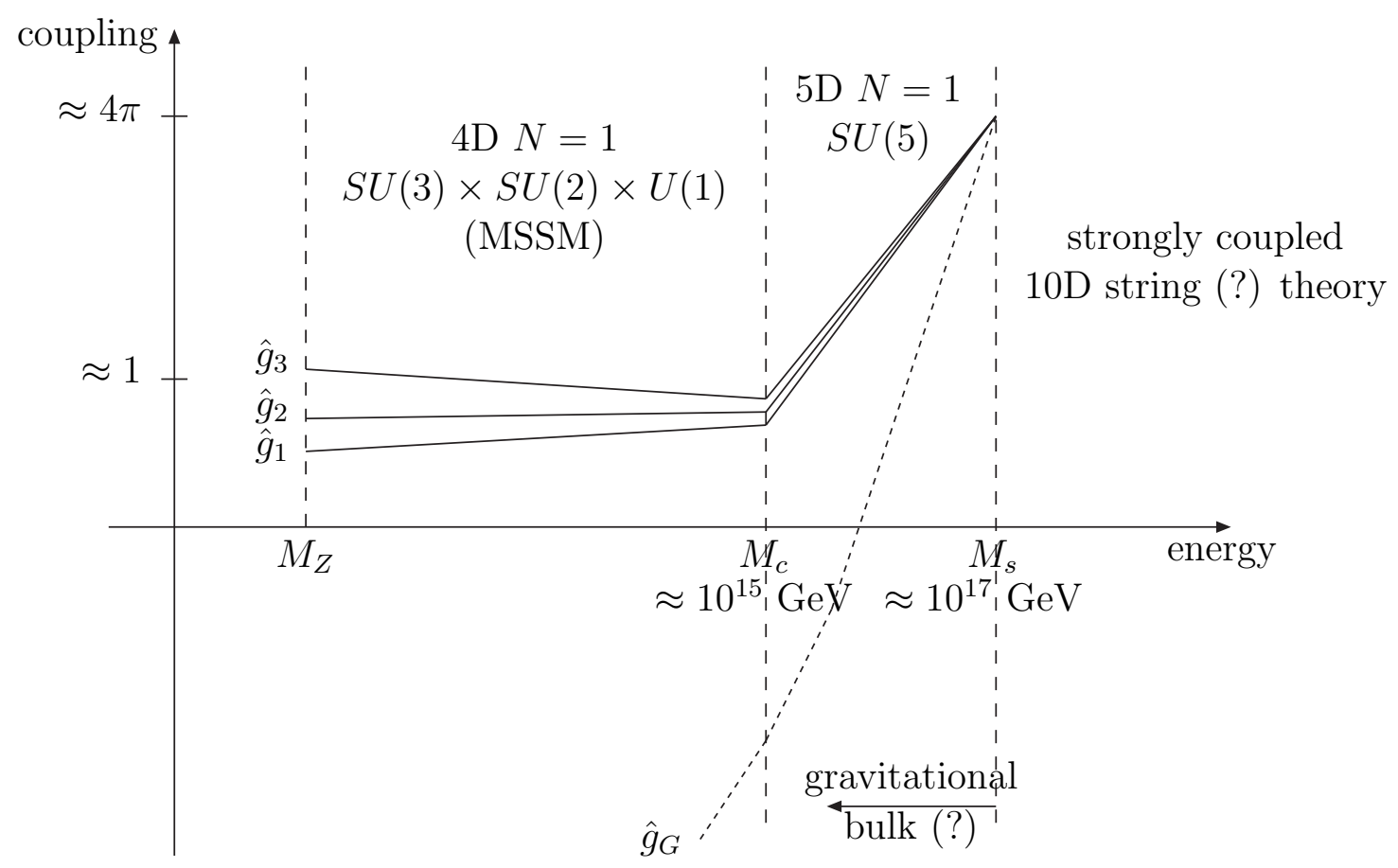

Figure 6: The energy dependence of the strengths of the gauge interactions.

effects of the 3-2-1 defect. Unification finally occurs at the fundamental scale. It is well known that a unification with two mass scales, such as $M_{c}$ and $M_{s}$, leads to a loss of predictivity of the low energy gauge couplings, since they depend on the extra parameter $M_{s} / M_{c}$. We overcome this by assuming that the theory at $M_{s}$ is strongly coupled, so that this mass ratio is predicted [2]:

$$
\left(\frac{M_{s}}{M_{c}}\right)^{d} \approx \frac{16 \pi^{2}}{C g^{2}\left(M_{c}\right)},
$$

where $C$ is a group theory Casimir; for example $C \approx 5$ for $G=S U(5)$. Given the rapid growth in the gauge couplings above $M_{c}$, and the strong coupling of the theory at $M_{s}$, one may wonder whether the unification can be reliably computed. Remarkably, however, the framework turns out to be extremely predictive. The strong coupling requirement allows us to reliably estimate the size of non-unified corrections from unknown ultraviolet physics, and the precise prediction for the low energy QCD coupling is obtained as long as the volume of the 3-2-1 defects is sufficiently small $[2,15]$. The uncertainties in the estimate of Eq. (3), for example from powerlaw corrections to gauge couplings [16], are also well under control and have little effect on the prediction [2].

In our scheme, the leading power correction to the gauge couplings, which is not a calculable quantity in the effective field theory, is universal and thus does not contribute to the low 


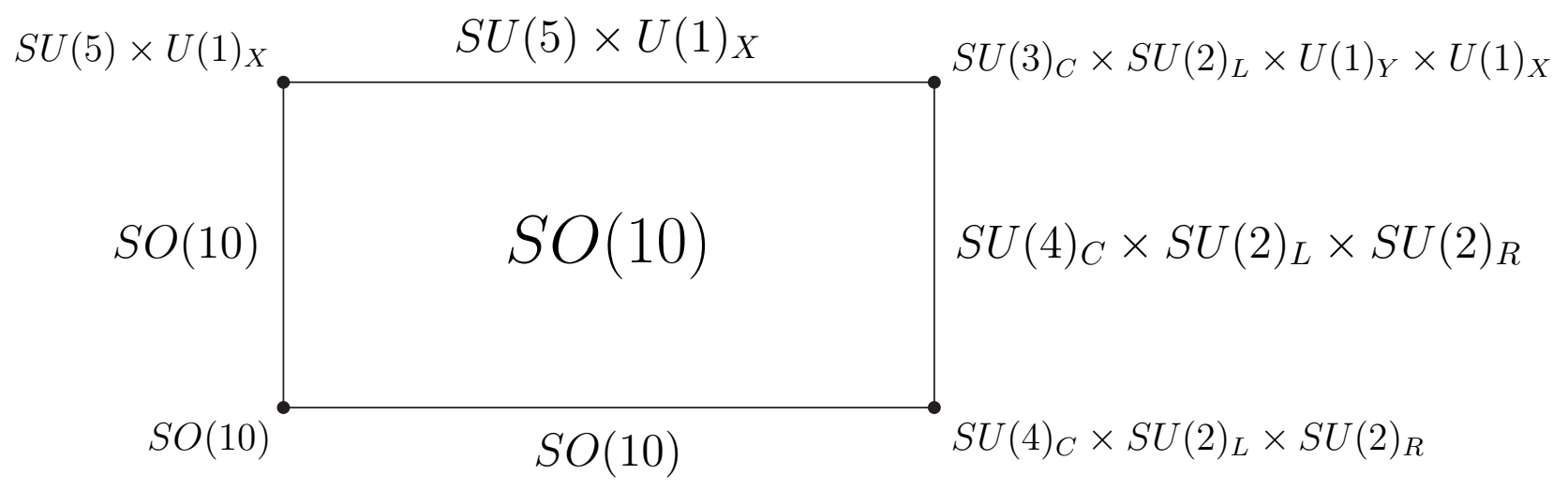

Figure 7: A 2D bulk with $S O(10)$ gauge symmetry allows for an interesting set of defects. There are two 1D defects, one having the usual $S U(5)$ unified gauge symmetry and the other having the left-right $S U(4)_{C} \times S U(2)_{L} \times S U(2)_{R}$ symmetry introduced by Pati and Salam. At the intersection of these two defects a point defect arises with the 3-2-1 symmetry of the standard model, together with an extra $U(1)_{X}$ symmetry.

energy prediction $[1,17]$. The relative running of the gauge couplings, which is crucial for the prediction, is then reliably computed if the volume of the defects is sufficiently small - that is, if the defects can effectively be viewed as points in the bulk: $d^{\prime}=0$. In such a setup, the low energy QCD coupling can be predicted in terms of the geometry of the bulk and the boundary conditions imposed on the bulk fields $\phi(y)$ :

$$
\alpha_{s}=\alpha_{s}(d, \text { geometry, boundary conditions, } \phi(y)) \text {. }
$$

As we go to a higher dimensional bulk, many more possibilities open up for the structure of gauge symmetry breaking by boundary conditions. A variety of defects can be incorporated. An example of a $G=S O(10)$ theory in a 2D bulk [18] is shown in Figure 7.

There are two 1D defects: a line where the gauge symmetry is $S U(5) \times U(1)_{X}$ and a line where the gauge symmetry is the Pati-Salam subgroup $S U(4)_{C} \times S U(2)_{L} \times S U(2)_{R}$. The intersections of these lines gives a point defect where the reduced symmetry is that of the standard model, augmented by an extra $U(1)_{X}$. Other $S O(10)$ models with a $2 \mathrm{D}$ bulk are also possible [19, 18], but generically it is hard, though not impossible [20], to reduce the rank by boundary conditions.

The prediction from gauge coupling unification differs in all these variations. As shown in section 3, the minimal $S U(5)$ theory with a single extra dimension agrees most precisely with data, and this suggests that in theories with higher gauge unification, such as $S O(10)$ in $6 \mathrm{D}$, one dimension of the box is larger than the others [21]. 


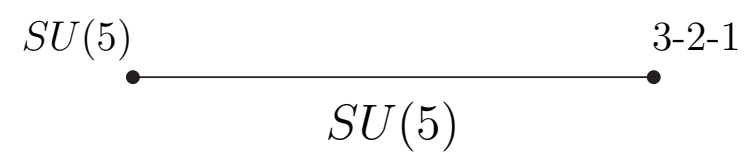

Figure 8: The minimal structure: $S U(5)$ gauge symmetry in a 1D bulk with a single 3-2-1 point defect.

\subsection{Split multiplets}

If the unified multiplet $H$ which contains the Higgs doublet of the standard model is described by a bulk field $H(y)$, then the "machine" of Figure 5 will automatically lead to a splitting of order $1 / R$ between the $S U(2)$ doublet component, $h_{2}$, and other components. The "doublettriplet splitting" puzzle of 4D unified theories is gone: indeed, mass splittings between components of bulk multiplets are unavoidable! This phenomenon has been known since the mid 80s [22, 23]. However, the implementation of this for the Higgs multiplet is not obvious there are many possible geometries and boundary conditions; and with supersymmetry in the higher dimensional bulk, as needed for gauge coupling unification, the field $H(y)$ contains many more superpartners than the 4D case. In 2000, Kawamura discovered an extremely elegant solution [24]: he studied a $G=S U(5)$ theory in 5D and constructed boundary conditions which broke the gauge symmetry to $3-2-1$ such that only the massless modes of $H(y)$ were the Higgs doublets and their usual 4D superpartners. The colored triplet components, whose exchange leads to proton decay, and all the 5D superpartners were found to only have massive modes.

In our language of defects the geometry following from his boundary conditions appears almost trivially simple: a 1D $S U(5)$ bulk, having a 3-2-1 defect at one boundary but not at the other, as shown in Figure 8. This case was also illustrated in Figures 2 and 4.

One might object that the whole idea of geometrical defects in gauge transformations is ugly, destroying the beauty of complete symmetry in the underlying theory broken only spontaneously by a dynamical choice of the vacuum. It is our contention that nature appears to prefer such defects, and the first hint of this was the understanding of the light Higgs doublets given by Kawamura.

\subsection{Proton decay}

In 4D supersymmetric $S U(5)$ grand unified theories, the two Higgs doublets, $h_{2}, \bar{h}_{2}$ are accompanied by their $S U(5)$ partners $H_{3}, \bar{H}_{3}$, which are color triplets. The exchange of the heavy colored Higgs fermions yields a proton decay amplitude at dimension five via the diagram shown in Figure 9. The cross on the internal line represents the Dirac mass that couples the fermions 


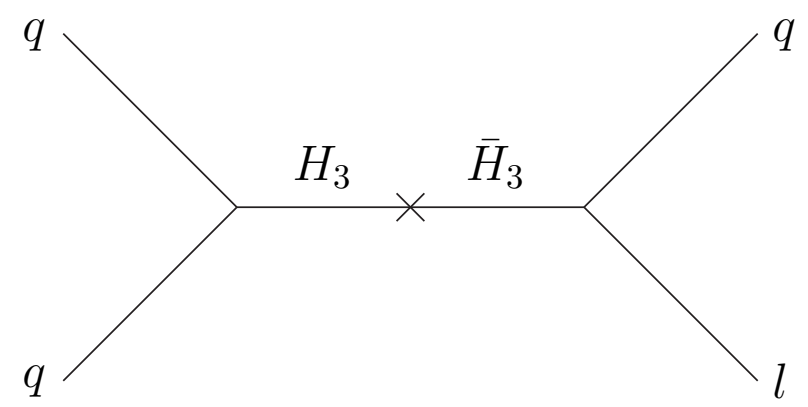

Figure 9: Baryon number violation is generated in 4D supersymmetric unified theories via the exchange of massive colored Higgs triplets.

in $H_{3}$ and $\bar{H}_{3}$. In the minimal theory this is the only way these fermions can get heavy, and the model is excluded by the resulting large amplitude for proton decay [10].

What happens in higher dimensional unified theories with symmetry breaking defects? At first sight the situation looks very bad: although we understand why the Higgs triplets are heavy and the Higgs doublets are light, the mass of the Higgs triplets will be determined by geometry and will be of the order of the compactification scale, $1 / R$. From gauge coupling unification we do not expect this to be large enough to avoid disastrous proton decay from $H_{3}$ fermion exchange. The origin of the masses of the modes in the KK towers is easily understood by considering the wavefunctions of the particles in a box with appropriate boundary conditions. For example, for the 5D $S U(5)$ theory, the curves of Figure 4 can be reinterpreted, with the solid curves being the wavefunction of the Higgs doublet modes and the dotted curves being the wavefunctions of the Higgs triplet modes. Since $E^{2}=p_{x}^{2}+p_{y}^{2}$, the momentum $p_{y}$ is interpreted as a mass in $4 \mathrm{D}$, so that the only massless mode is that of the Higgs doublet having the flat wavefunction. All the Higgs triplet modes have non-zero $p_{y}$ because boundary conditions force their wavefunctions to vanish at the 3-2-1 defect, and the corresponding $4 \mathrm{D}$ fields have masses of order $1 / R$. In the minimal theory discussed in the next section $1 / R \approx 10^{15} \mathrm{GeV}$, which is less than the unified mass scale in the conventional $4 \mathrm{D}$ theory. Hence one might expect a very large proton decay amplitude.

In $5 \mathrm{D}$, the form of the mass terms for the $H_{3}$ and $\bar{H}_{3}$ fermions is dictated by the higher dimensional spacetime symmetry of the bulk. Since the smallest fermion representation in 5D is a Dirac fermion, both $H_{3}$ and $\bar{H}_{3}$ fermions are accompanied by their conjugated fermions, $H_{3}^{c}$ and $\bar{H}_{3}^{c}$. The $5 \mathrm{D}$ kinetic terms for these fermions contain $H_{3} \partial_{y} H_{3}^{c}$ and $\bar{H}_{3} \partial_{y} \bar{H}_{3}^{c}$. From the viewpoint of $4 \mathrm{D}$ the masses arise from $\partial_{y}$, so that the Dirac mass for $H_{3}$ couples it to $H_{3}^{c}$ rather than to $\bar{H}_{3}$. The cross in the diagram of Figure 9 does not exist; $\bar{H}_{3}$ must be replaced by $H_{3}^{c}$. On the other hand, an $R$ symmetry arising from higher dimensional supersymmetry forbids 
any coupling of $H_{3}^{c}$ to quarks and leptons. Hence, we find that the proton decay amplitude from the exchange of the color triplet Higgs fermions necessarily vanishes in higher dimensional unified theories [1].

We have shown that the absence of all proton decay from operators in the low energy theory of dimension four or five is guaranteed by an $R$ symmetry [1, 2]. Hence the leading contribution will come at dimension six from the exchange of the heavy $X$ gauge bosons. This depends sensitively on the mass of these gauge bosons, which is also given by $1 / R$. The precise value of $1 / R$ is model dependent, and we will return to this issue in section 3 .

\subsection{Quark-lepton mass relations}

So far we have assumed that particles are free to propagate throughout the volume of the bulk. However, it may be that some particles are restricted to subspaces of the bulk. For example, in the box of Figure 3, quarks and leptons could be chosen to propagate in the entire 3D bulk, on a given 2D surface, a 1D line, or they may even be restricted to a point. A quark or lepton which propagates on a defect with lower gauge symmetry will only feel this lower symmetry: it will not live in a multiplet of the higher gauge symmetry of the full bulk. It would therefore seem less attractive to place quarks and leptons precisely on a 3-2-1 defect, since one would lose the immediate understanding of the gauge quantum numbers of a generation given by the higher gauge symmetry. ${ }^{1}$

What distinguishes one generation from another? Could it be that they propagate in differing numbers of dimensions in the bulk? This is an attractive idea because it leads to a geometrical understanding of the hierarchy between the masses of the generations. The quark and lepton masses arise from Yukawa couplings, but these interactions are forbidden by supersymmetry in dimensions higher than 4 . Hence the Yukawa coupling between fermion $\psi_{i}$ and fermion $\psi_{j}$ must be located at a point $y_{0}$ on the surface of the box where the higher dimensional Lorentz and supersymmetry is broken: $\mathcal{L}_{\text {Yukawa }}=\delta^{d}\left(y-y_{0}\right) \psi_{i} \psi_{j} H(y)$. Since the quarks and leptons that we observe are zero mass modes in the box, they have flat wavefunctions with normalizations $1 / \sqrt{V}$, where $V$ is the volume of the subspace of the bulk in which they propagate. Integrating over the volume of the bulk to get the equivalent $4 \mathrm{D}$ theory then leads to an entry in the fermion mass matrix

$$
m_{i j} \propto \frac{1}{\sqrt{V_{i} V_{j}}}
$$

providing both subspaces cover the point $y_{0}$.

The above relation implies that the heaviest fermions propagate in only a small subspace

\footnotetext{
${ }^{1}$ On the other hand, placing the Higgs on a 3-2-1 defect is an alternative way to understand the absence of a color triplet Higgs in the low energy theory [25].
} 
of the bulk. This makes it likely that their Yukawa coupling is located far from the G-breaking defects, so that they will exhibit unified mass relations between quarks and leptons. On the other hand, lighter fermions must live in a larger subspace of the bulk, and are more likely to propagate past the defects. Yukawa couplings located on these defects will destroy any unified mass relations. Unified mass relations between quarks and leptons are expected only for the heaviest generation $[2,26,27,15]$.

To conclude: higher dimensional grand unified theories with symmetry breaking defects offer a remarkable possibility. The conventional successes of grand unified theories (quark-lepton gauge quantum numbers, gauge coupling unification, and mass relations for heavy fermions) can be retained as phenomena of the symmetrical bulk, while conventional difficulties (mass splitting between $h_{2}$ and $H_{3}$, proton decay, and light fermion mass relations) are automatically resolved as phenomena of the defects.

\section{The Minimal Model}

Up to now we have concentrated on the conceptual advantages of higher dimensional unified theories. We now show that these theories are remarkably predictive if they are valid over a large energy range, i.e. if $M_{s} / M_{c}$ is large, and present a minimal model [2] which is highly successful in describing physics over a wide energy interval between $M_{s}$ and $M_{c}$.

\subsection{Preferred by gauge coupling unification}

The numerical test for any unified theory is gauge coupling unification, so we intend to use this as a tool to guide us in searching for a particular higher dimensional geometry. Recall from Figure 1: while conventional supersymmetric unification does well, it is not perfect. We have also seen from Figure 5 that the box and boundary conditions are a "machine" for creating KK towers of particles. Could the difference between the central value of the conventional prediction, $\alpha_{s}\left(M_{Z}\right)=0.130$, and experiment, $\alpha_{s}\left(M_{Z}\right)=0.117$, be due to the virtual effects of these KK modes? If so, are there any geometries that are simple enough that they are numerically predictive?

We have performed a detailed study of supersymmetric theories with $d$ extra dimensions with equal radii. For $d \geq 3$ there are no corrections from the KK modes because of cancellations forced by the large amount of supersymmetry in higher dimensions. For $d=1,2$ the leading logarithmic correction is [2]

$$
\delta \alpha_{s} \simeq-\frac{6}{7 n \pi} \alpha_{s}^{2} \ln \frac{M_{s}}{M_{c}}
$$

where $n=2$ for $d=1$, and for $d=2$ it is a positive integer $\geq 2$, describing the geometry of

the box. Here, $\delta \alpha_{s}$ is defined by the difference of our prediction, $\alpha_{s}^{\mathrm{KK}}$, and the conventional 


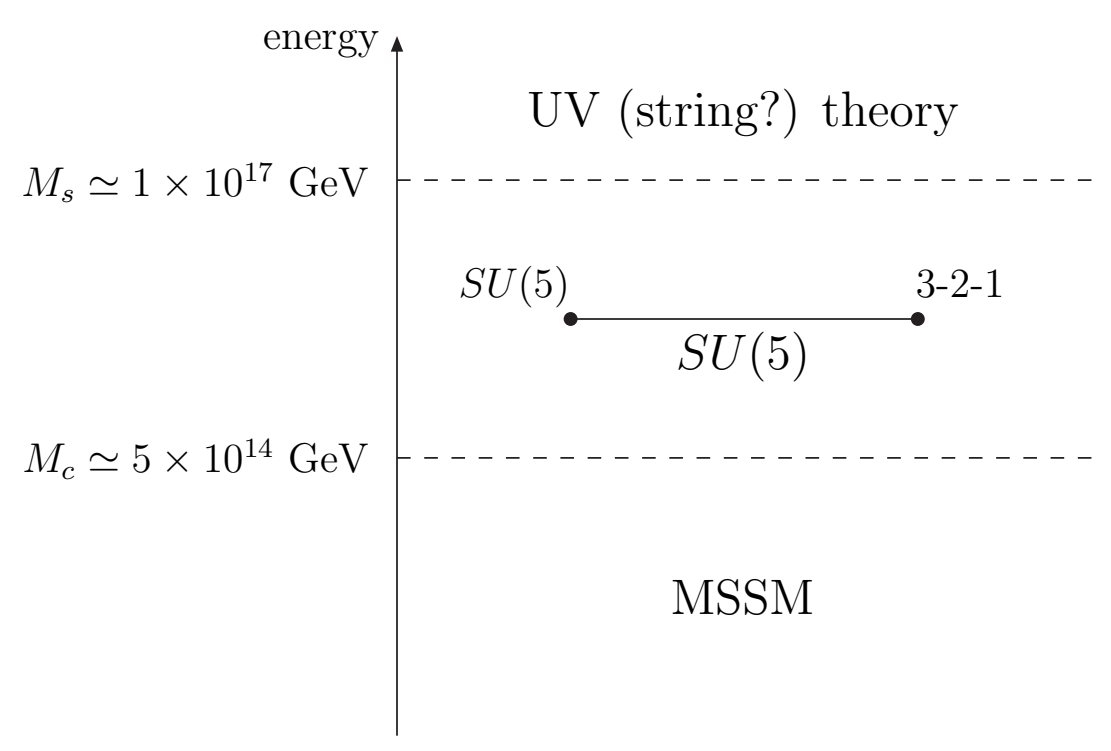

Figure 10: The scheme preferred by gauge coupling unification: the minimal supersymmetric standard model (MSSM) is the effective theory up to $M_{c} \approx 5 \times 10^{14} \mathrm{GeV}$, while the effective theory for the next factor of 200 in energy is the minimal $5 \mathrm{D} S U(5)$ theory with a single 3-2-1 defect.

prediction, $\alpha_{s}^{\mathrm{SGUT}}: \delta \alpha_{s}=\alpha_{s}^{\mathrm{KK}}-\alpha_{s}^{\mathrm{SGUT}}$. This result applies only if the Higgs doublets propagate in the bulk, and are not contained in the vector multiplet; otherwise the sign of the correction is changed, increasing the discrepancy with data. While this result is very simple, and the sign is very encouraging, apparently we cannot evaluate it numerically because of the unknowns $M_{s} / M_{c}$ and $n$. However, using our assumption that the theory is strongly coupled at the fundamental scale, $M_{s} / M_{c}$ can be estimated as in Eq. (3). From this we discover that for most values of $(d, n)$ the correction $\left|\delta \alpha_{s}\right|$ is too small to give perfect agreement with data. Only in the case that it is maximized does the central value of the theoretical prediction agree with data, and this occurs for the simplest case of a single extra dimension, $d=1$ (hence $n=2$ ). In this case the unified gauge group should be $S U(5)$, since larger unified groups cannot be broken by boundary conditions in a single extra dimension to $3-2-1-G^{\prime}$, so that gauge coupling unification would depend on further symmetry breaking and predictivity would be lost. These considerations lead to the effective theory below $M_{s}$ as given in Figure 10.

Note that we are able to go much further than conventional supersymmetric unification which simply identifies a single scale $M_{u} \simeq 2 \times 10^{16} \mathrm{GeV}$ as the threshold for unified physics. We can determine both the compactification scale, $M_{c} \simeq 5 \times 10^{14} \mathrm{GeV}$, and the scale of strong coupling, $M_{s} \simeq 1 \times 10^{17} \mathrm{GeV}$, and consequently the masses of all the $\mathrm{KK}$ modes of gauge 


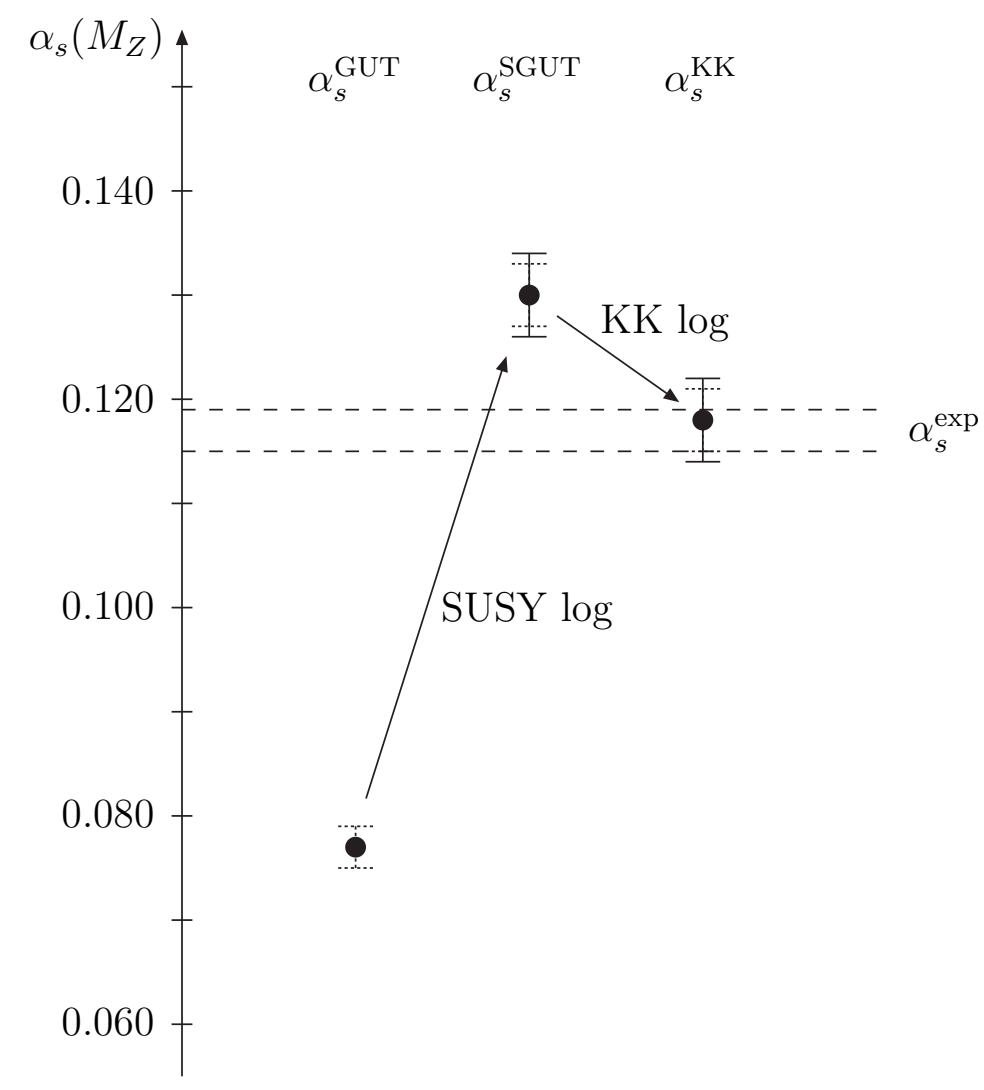

Figure 11: The predictions for $\alpha_{s}\left(M_{Z}\right)$ in the three frameworks: non-supersymmetric grand unification $\alpha_{s}^{\mathrm{GUT}}$, supersymmetric grand unification $\alpha_{s}^{\mathrm{SGUT}}$, and higher dimensional grand unification $\alpha_{s}^{\mathrm{KK}}$. Solid error bars represent the threshold corrections from the superpartner spectrum. Dotted error bars for $\alpha_{s}^{\mathrm{GUT}}$ and $\alpha_{s}^{\mathrm{SGUT}}$ represent threshold corrections from the unified scale corresponding to a heavy $\mathbf{5}+\overline{\mathbf{5}}$ representation with unit logarithmic mass splitting between doublets and triplets. The dotted error bar for $\alpha_{s}^{\mathrm{KK}}$ is the theoretical uncertainty (other than from superpartner masses) for our theory, as estimated in Ref. [2]. 
bosons, Higgs and matter in this energy interval. ${ }^{2}$ Apart from discrete choices, such as the location of the quarks and leptons of the various generations, we determine the entire effective theory that is valid over an energy range spanning a factor of 200.

In Figure 11 we show the effect of the logarithm from the KK modes in the minimal model the prediction is strikingly successful. Because the higher dimensional theory is valid over such a large energy interval, the uncertainties to this correction are small, as shown in the figure. The dominant uncertainty in the prediction now comes from the supersymmetric threshold, which ultimately will be fixed by data.

At energies approaching $M_{s}$, our 5D effective theory will break down. It could be that a higher dimensional structure emerges, such as that of Figure 7, with the vertical dimension much less than the horizontal one. At this scale it may be possible to interpret the Higgs as arising from a vector multiplet, for example as a component of the higher dimensional gauge field $[21,26]$.

\subsection{Yukawa coupling unification}

In section 2 we have argued that heavier quarks and leptons should propagate in subspaces of the bulk with lower dimension. In the minimal theory the heavy third generation should reside at a boundary of the fifth dimension. To retain the $S U(5)$ understanding of quantum numbers, this should be the "SU(5) boundary" rather than the "3-2-1 boundary", giving the usual tree-level $S U(5)$ mass relation: $m_{b}=m_{\tau}$. In conventional unified theories the corrections to this relation from running of the Yukawa couplings can be accurately computed, but there are also corrections from both unknown physics at the unified scale, and from supersymmetric corrections at the weak scale. In our theory the unified physics is known, and hence we can compute the corrections at the unified scale. The resulting correction to the prediction for the $b$ quark mass is [3]

$$
\frac{\delta m_{b}}{m_{b}} \simeq-\frac{5\left(4 g^{2}-y_{t}^{2}\right)}{112 \pi^{2}} \ln \frac{M_{s}}{M_{c}} .
$$

As in Eq. (6) for the radiative corrections to $\alpha_{s}$, the sign improves agreement with data. However, unlike gauge coupling unification, the prediction for the $b$ quark mass receives large supersymmetric corrections [28], and hence we leave the comparison with data until section 4 where we incorporate supersymmetry breaking.

Since the minimal theory has only a single extra dimension, all of flavor cannot be understood in terms of volume factors. Nevertheless, some of the quarks and leptons will have suppressed masses because they propagate in the bulk, and these light fermions will not exhibit

\footnotetext{
${ }^{2}$ Here and below, $M_{c}$ represents the length scale of the extra dimension, $M_{c}=(\pi R)^{-1}$, which is denoted as $M_{c}^{\prime}$ in Refs. [2, 3].
} 


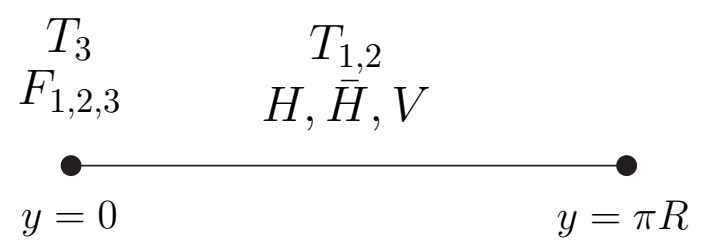

Figure 12: Locations of $S U(5)$ matter, Higgs and gauge multiplets in the fifth dimension.

unified mass relations. For example, if the right-handed up quark, $u$, and electron, $e$, reside in the bulk they will be the zero modes of a 10-dimensional representation of $S U(5): T(u, e)$, where the zero modes are written in parenthesis. However, in this case a light quark doublet $q$ does not arise from this multiplet; rather it arises from another 10-plet having boundary conditions with opposite sign: $T^{\prime}(q)$. This may appear to be a step backwards - the light quarks and leptons are not as unified as in conventional $S U(5)$. However, it can also be viewed as a virtue: disastrous unified mass relations for light matter are avoided, while the understanding for quark and lepton quantum numbers is preserved. To see the absence of a mass relation, consider the fermion Yukawa couplings in the case where the right-handed down quark, $d$, and the lepton doublet, $l$, are unified into the same 5-dimensional multiplet: $F(d, l)$. In this case the mass terms for the electron and down quark arise from two distinct interactions $T F \bar{H}$ and $T^{\prime} F \bar{H}$, respectively, so there is clearly no $S U(5)$ relation between these masses.

One plausible possibility is that the lightest two generations of 5 -plets $F_{1,2}$ are located on the boundary, while the corresponding 10-plets $T_{1,2}$ are bulk modes [3], as depicted in Figure 12. There is no flavor distinction between the three $F$, so that large neutrino mixing angles are expected. Small neutrino masses can be understood by introducing right-handed neutrinos $N_{1,2,3}$, through the conventional see-saw mechanism [29]. Flavor hierarchies in masses and mixings arise from the $T$, both from volume factors $[2,26,27,15]$ and from distortions of the wavefunctions caused by bulk mass terms [30]. Potential brane-localized anomalies are canceled by a certain bulk term made out of the bulk gauge field [31]. This gives a larger hierarchy for up-type quark masses (from $T T$ ) than for down-type and charged lepton masses (from $T F$ ). The absence of unified relations amongst the lighter two generation quarks and leptons is entirely due to $T_{1,2}$ (which really represent $T_{1,2}(u, e)$ and $T_{1,2}^{\prime}(q)$ ).

\subsection{Predictions for proton decay}

In section 2 we saw that higher dimensional theories possess a $U(1)_{R}$ symmetry that forbids proton decay from operators of dimension five resulting from the exchange of the colored triplet Higgs. This $R$ symmetry arises from a phase rotation of the coordinates of superspace - it is 
an unavoidable consequence of supersymmetry in the higher dimensional bulk. In 4D supersymmetric theories one must impose a discrete symmetry by hand to avoid baryon- and leptonnumber violation at the weak scale arising from the superpotential interactions $u d d, q d l, l l e$ and $l h$. In higher dimensional theories this parity can be understood as a subgroup of the continuous $R$ symmetry. Of course, one cannot prove that these interactions must be absent after all there might be an $R$ symmetry breaking defect on the boundary; however, we can say that in higher dimensional theories there is a very plausible origin for the conventional discrete symmetry.

Do these theories predict proton stability? No - in general the heavy gauge bosons of the unified theory can induce proton decay, and hence we must study the masses and couplings of these gauge bosons in models of interest. In the $5 \mathrm{D} S U(5)$ theory there is a KK tower of $X$ gauge bosons with the lightest mode having mass $1 / 2 R=\pi M_{c} / 2$. We have seen that a calculable weak mixing angle requires a large value of $M_{s} / M_{c}$, and therefore a small value for $M_{c}$. The observed values of the gauge couplings strongly suggest that $M_{s}$ is the scale of strong coupling, so that $M_{c} \simeq 5 \times 10^{14} \mathrm{GeV}$. Hence this gauge boson has about the same mass as in the $4 \mathrm{D}$ Georgi-Glashow $S U(5)$ theory. It appears that we have come around a full circle, and are excluded, like the non-supersymmetric $S U(5)$ theory, by searches for proton decay. However, we have not yet investigated the couplings of $X$ in the 5D theory. We have argued that since the electron and up quark are so light they should reside in the bulk; thus these states are described by two 10-plets $T_{1}(u, e)$ and $T_{1}^{\prime}(q)$. This means that the conventional interactions of the $X$ boson, $q^{\dagger} u X, e^{\dagger} q X$ are not generated by the bulk gauge interactions of the $5 \mathrm{D}$ theory, at least for the lightest generation, and hence in the absence of CKM mixing between the generations the proton would be stable [1]. The mode expected from CKM mixing is $p \rightarrow K^{+} \bar{\nu}$, but the rate is now highly dependent on the flavor structure of the theory [15, 32], and while the rate is no longer too large, it is not guaranteed to be in reach of future detectors.

Remarkably there is an additional source for the $q^{\dagger} u X, e^{\dagger} q X$ interactions. They result from a boundary localized contribution to the gauge interactions, and therefore have a size which is suppressed relative to the usual gauge coupling by the volume factor $M_{c} / M_{s}$. The proton lifetime cannot be precisely predicted since the boundary gauge interaction involves a dimensionless coupling that is not predicted. If this coupling is of order unity then $\tau_{p} \approx$ $10^{34}$ years, with comparable branching ratios for the decay modes $e^{+} \pi^{0}, \mu^{+} \pi^{0}, e^{+} K^{0}, \mu^{+} K^{0}, \pi^{+} \bar{\nu}$ and $K^{+} \bar{\nu}$ [3]. The most promising discovery mode is $e^{+} \pi^{0}$. A large mixing angle between $F_{1}$ and $F_{2}$ implies that the $e^{+} \pi^{0}$ and $\mu^{+} \pi^{0}$ modes have comparable branching ratios and that

$$
\frac{\Gamma\left(p \rightarrow \mu^{+} \pi^{0}\right)}{\Gamma\left(p \rightarrow e^{+} \pi^{0}\right)} \simeq \frac{\Gamma\left(p \rightarrow e^{+} K^{0}\right)}{\Gamma\left(p \rightarrow \mu^{+} K^{0}\right)},
$$

independent of the sizes of hadronic matrix elements [3]. This analysis for proton decay depends 


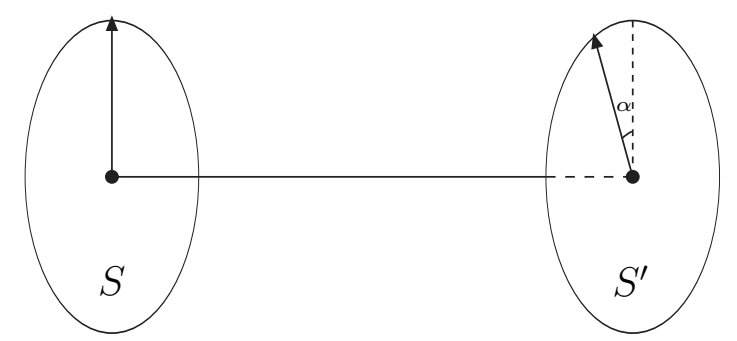

Figure 13: The breaking of supersymmetry by the misalignment of boundary defects.

on matter location, but is completely independent of supersymmetry breaking.

\section{Supersymmetry Breaking}

Theories with weak scale supersymmetry will lead to a plethora of new particles and couplings to be measured in the TeV domain. The supersymmetry breaking interactions, like the gauge and Yukawa interactions, may encode information about the unified theory [33]. For this to happen they must remain as local interactions up to the unification scale - we say that the messenger scale of supersymmetry breaking must be at least as large as the unification scale. If this happens in higher dimensional theories, then the soft supersymmetry breaking operators will provide a window on the physics of the bulk [3]. In particular, the flavor symmetry of the bulk $S U(5)$ gauge interactions $U(3)_{T} \times U(3)_{F}$ will be modified by the locations of the three $T$ and three $F$ fields. This will lead to non-universal squark and slepton masses, and to flavor changing neutral currents from superpartner exchange, allowing experiment to probe the geometry and matter locations in the bulk.

Such signals will depend on the mechanism for supersymmetry breaking. In this section we discuss the possibility that boundary conditions in the bulk break supersymmetry as well as the unified gauge symmetry [34]. In the minimal 5D $S U(5)$ model there is a unique way to accomplish such a breaking [35]. In the 5D bulk there are two independent supersymmetry transformations and they form a doublet of the $S U(2) R$ symmetry. However, the boundaries are four dimensional and can support only a single supersymmetry. Thus the boundaries can be viewed as defects in the space of supersymmetry transformations, and the key question is whether the two supersymmetries, $S$ and $S^{\prime}$, respected by the two boundaries are the same or not, as illustrated in Figure 13. In the limit that the relative orientation angle $\alpha$ vanishes, $S^{\prime}=S$ and the single supersymmetry $S$ is preserved everywhere and must therefore be a symmetry of the low energy 4D theory. On the other hand, if there is a misalignment of the two defects characterized by a non-zero value of $\alpha$, then supersymmetry is broken in the low energy theory, 
with the supersymmetry breaking mass scale given by $\tilde{m}=\alpha M_{c}$. The appearance of the continuous parameter $\alpha$ describing the defects represents an important difference compared with the case of $S U(5)$ gauge symmetry breaking, where the breaking scale is necessarily $M_{c}$. The parameter $\alpha$ can also be viewed as the vacuum condensation of a component of the higher dimensional gravitational supermultiplet [36], implying that the supersymmetry breaking is spontaneous and the hierarchy of the scales, $\tilde{m} \ll M_{c}$, can naturally be obtained.

The tree-level form of the supersymmetry breaking soft operators is very simple [3]. All bulk superpartners have mass $\tilde{m}$, all boundary-located superpartners are massless, and the size of the trilinear scalar interaction is $\tilde{m}, 2 \tilde{m}$ or $3 \tilde{m}$, counting the number of bulk scalars:

$$
\begin{aligned}
\mathcal{L}_{\text {soft }}= & -\frac{1}{2}(\tilde{m} \lambda \lambda+\text { h.c. })-\tilde{m}^{2} h^{\dagger} h-\tilde{m}^{2} \tilde{f}_{B}^{\dagger} \tilde{f}_{B} \\
& +\left(y_{f} \tilde{m} \tilde{f}_{b} \tilde{f}_{b} h+2 y_{f} \tilde{m} \tilde{f}_{B} \tilde{f}_{b} h+3 y_{f} \tilde{m} \tilde{f}_{B} \tilde{f}_{B} h+\text { h.c. }\right),
\end{aligned}
$$

where $\lambda, h, \tilde{f}_{B}$ and $\tilde{f}_{b}$ collectively represent the gauginos, two Higgs doublets, squarks/sleptons in the bulk and squarks/sleptons on the brane, respectively, and $y_{f}$ is the value of the corresponding Yukawa coupling. Since supersymmetry breaking effects from boundary conditions are shut off above the compactification scale, the soft supersymmetry breaking masses in Eq. (9) must be regarded as the running mass parameters at the compactification scale $M_{c}$. Incidentally, this type of theory can also generate the weak-scale $\mu$ and $B$ terms naturally through the vacuum readjustment mechanism [37].

Applying these results to the minimal model yields two immediate consequences: first the flavor changing neutral current effects induced by superpartner exchange are too large unless the squarks (sleptons) of the first two generations are degenerate. This means that $T_{1}$ and $T_{2}$ must have the same location, and similarly for $F_{1}$ and $F_{2}$. When coupled with constraints from proton decay and from fermion mass relations, the location assignments of Figure 12 become unique. Hence, we are now able to predict that the neutrino mixing angles are large. Secondly the Higgs potential and superpartner masses are determined in terms of only three parameters $\tilde{m}, \mu$ and $B$. After fixing the $Z$ mass the two independent parameters can be taken to be $\tilde{m}$ and $\tan \beta$, the ratio of the two Higgs vacuum expectation values. The entire superpartner and Higgs spectrum is shown in Table 1 for two representative values of these parameters. The mass of the lightest Higgs boson, $h$, includes one-loop radiative corrections from top quarks and squarks. The sign and large size of the relevant scalar trilinear of Eq. (9) makes these corrections large and the resulting Higgs boson becomes relatively heavy. The squarks and sleptons from $T_{3}$ and $F_{1,2,3}$ are relatively light as they reside on the boundary and do not get mass at tree level. For example, this makes $\tilde{l}$ lighter than $\tilde{e}$. The two lightest superpartners are the scalar tau and the bino. Either could be the LSP, and hence collider signals involve either stable charged particle tracks or missing transverse energy [3]. 


\begin{tabular}{|c|c|c|}
\hline $\begin{array}{c}\tilde{m} \\
\tan \beta\end{array}$ & $\begin{array}{c}300 \\
5\end{array}$ & $\begin{array}{c}400 \\
10\end{array}$ \\
\hline$\overline{\tilde{g}}$ & 699 & 911 \\
\hline$\tilde{\chi}_{1}^{ \pm}$ & 251 & 334 \\
\hline$\tilde{\chi}_{2}^{ \pm}$ & 427 & 531 \\
\hline$\tilde{\chi}_{1}^{0}$ & 130 & 175 \\
\hline$\tilde{\chi}_{2}^{0}$ & 251 & 334 \\
\hline$\tilde{\chi}_{3}^{0}$ & 417 & 518 \\
\hline$\tilde{\chi}_{4}^{0}$ & 422 & 528 \\
\hline$\tilde{\tilde{q}}$ & 701 & 915 \\
\hline$\tilde{u}$ & 675 & 880 \\
\hline$\tilde{d}$ & 602 & 780 \\
\hline$\tilde{l}$ & 209 & 277 \\
\hline$\tilde{e}$ & 317 & 422 \\
\hline$\tilde{t_{1}}$ & 425 & 547 \\
\hline$\tilde{t}_{2}$ & 619 & 780 \\
\hline$\tilde{b}_{1}$ & 563 & 727 \\
\hline$\tilde{b}_{2}$ & 601 & 774 \\
\hline$\tilde{\tau}_{1}$ & 106 & 126 \\
\hline$\tilde{\tau}_{2}$ & 214 & 280 \\
\hline $\bar{h}$ & 118 & 128 \\
\hline$A$ & 552 & 690 \\
\hline$H^{0}$ & 553 & 690 \\
\hline$H^{ \pm}$ & 558 & 695 \\
\hline$\alpha_{s}\left(M_{Z}\right)\{ \pm 0.003\}$ & 0.119 & 0.118 \\
\hline$m_{b}\left(M_{Z}\right)\{ \pm 0.10\}$ & 3.37 & 3.26 \\
\hline $\operatorname{Br}(\mu \rightarrow e \gamma)$ & $6 \times 10^{-12}$ & $8 \times 10^{-12}$ \\
\hline $\operatorname{Br}(\mu \rightarrow 3 e)$ & $4 \times 10^{-14}$ & $5 \times 10^{-14}$ \\
\hline $\mathrm{Cr}\left(\mu \rightarrow e ;{ }_{22}^{48} \mathrm{Ti}\right)$ & $4 \times 10^{-14}$ & $5 \times 10^{-14}$ \\
\hline $\operatorname{Br}(\tau \rightarrow \mu \gamma)$ & $1 \times 10^{-8}$ & $1 \times 10^{-8}$ \\
\hline
\end{tabular}

Table 1: Predictions for the superpartner spectrum, the Higgs spectrum, gauge and Yukawa unification, and lepton flavor violating processes. The predictions are for two representative values of $\tilde{m}$ and $\tan \beta$, and all masses are given in GeV. Mass eigenvalues are given for the gluino, $\tilde{g}$, the charginos, $\tilde{\chi}^{ \pm}$, the neutralinos, $\tilde{\chi}^{0}$, the squarks and sleptons of the third generation, $\tilde{t}_{1,2}, \tilde{b}_{1,2}$ and $\tilde{\tau}_{1,2}$, and the Higgs bosons, $h, A, H^{0}$ and $H^{ \pm}$. For the first two generations of squarks and sleptons the masses are shown for $\tilde{q}, \tilde{u}, \tilde{d}, \tilde{l}$ and $\tilde{e}$ and do not include contributions from electroweak $D$ terms. 


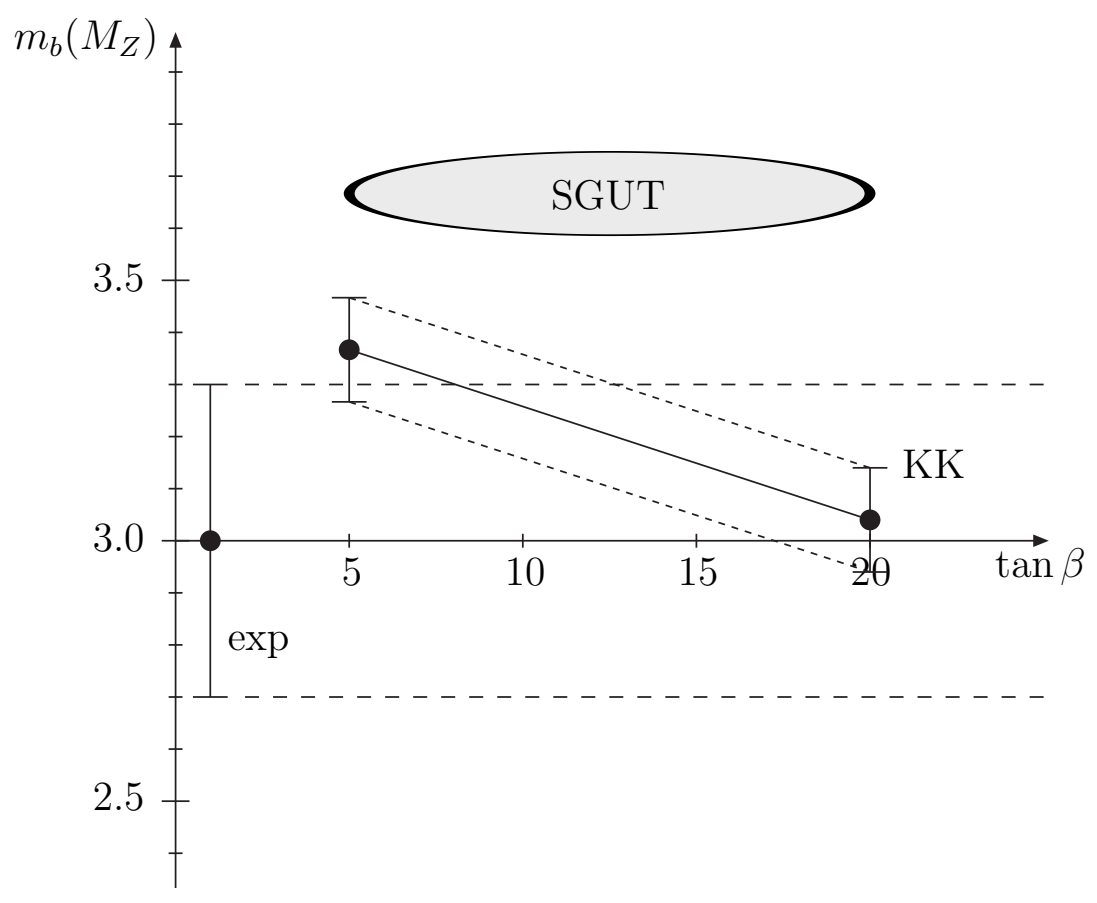

Figure 14: A comparison of the prediction of the $b$ quark mass from Yukawa unification with experiment. The prediction from conventional 4D unification, without supersymmetric threshold corrections is too large. In the minimal 5D $S U(5)$ theory, the unified corrections of Eq. (7) bring the prediction within $1 \sigma$ of the data. If supersymmetry is broken by boundary conditions, the superpartner corrections are linear in $\tan \beta$ and bring complete agreement with data. Values of $\tan \beta$ larger than about 20 are disfavored by lepton flavor changing processes. 
Now that the superpartner spectrum is known, the supersymmetric threshold correction for the $b$ quark mass prediction from Yukawa unification can be calculated. Since we are computing the correction to a dimensionless Yukawa coupling, $\tilde{m}$ drops out and the result depends only on $\tan \beta$ : the fractional correction to the $b$ quark mass is $-0.006 \tan \beta$ if the $\mu$ parameter is negative. The prediction for $m_{b}\left(M_{Z}\right)$ is shown in Figure 14, including the unified threshold corrections of Eq. (7). The supersymmetric threshold correction to the QCD coupling can also be calculated, and these predictions may be written in the compact form [3]

$$
\begin{aligned}
\alpha_{s}\left(M_{Z}\right) & =\left(0.1182-0.0030 \ln \frac{\tilde{m}}{400 \mathrm{GeV}}-0.0019 \ln \frac{M_{s} / M_{c}}{200}\right) \pm 0.003 \\
m_{b}\left(M_{Z}\right) & =\left(3.26-0.022(\tan \beta-10)-0.026 \ln \frac{M_{s} / M_{c}}{200}\right) \pm 0.1 \mathrm{GeV}
\end{aligned}
$$

where we have also included the finite correction to $\alpha_{s}\left(M_{Z}\right)$ from $M_{c}$, calculated using dimensional regularization [38].

The third generation squarks and sleptons are massless at tree level since they reside on the boundary, while the squarks and sleptons of the first two generations of $T$ have a tree level mass $\tilde{m}$ as they propagate in the bulk. This means that the $U(3)_{T}$ flavor symmetry is broken to $U(2)_{T}$ at tree level in the superpartner spectrum. The flavor changing effects triggered by this flavor symmetry breaking are expected to be larger than in conventional supersymmetric unification with gravity mediated supersymmetry breaking, where such flavor breaking occurs only through top quark radiative corrections $[33,39,40]$. The signals are particularly important in the lepton sector. By rotating to a mass eigenstate basis for charged leptons, while maintaining diagonal scalar mass-squared matrices, we can go to a basis where the lepton flavor violation appears only via a single new mixing matrix $W^{e}$ in the lepton-slepton-gaugino interaction:

$$
\mathcal{L}^{\mathrm{LFV}}=-\left(\sqrt{2} g^{\prime} e W^{e \dagger} \tilde{e}^{\dagger} \tilde{b}+\text { h.c. }\right),
$$

and in Higgs interactions. Here, $\tilde{b}$ represents the $U(1)_{Y}$ gaugino and

$$
W^{e}=R_{23}^{e} R_{12}^{e}=\left(\begin{array}{ccc}
c_{12}^{e} & -s_{12}^{e} & 0 \\
s_{12}^{e} c_{23}^{e} & c_{12}^{e} c_{23}^{e} & -s_{23}^{e} \\
s_{12}^{e} s_{23}^{e} & c_{12}^{e} s_{23}^{e} & c_{23}^{e}
\end{array}\right)
$$

where $c_{i j}^{e} \equiv \cos \theta_{i j}^{e}$ and $s_{i j}^{e} \equiv \sin \theta_{i j}^{e}$. Therefore, we find a remarkable result that all the lepton flavor violating processes are completely described by two angles, $\theta_{12}^{e}$ and $\theta_{23}^{e}$, as far as the charged lepton sector is concerned.

The gaugino exchange diagram for $\mu \rightarrow e \gamma$ is shown in Figure 15. Including other one-loop contributions, we find the branching ratio to be [3]

$$
\operatorname{Br}(\mu \rightarrow e \gamma) \simeq 3 \times 10^{-11}\left(\frac{200 \mathrm{GeV}}{\tilde{m}}\right)^{4}\left(\frac{\left|W_{\tau \mu}^{e}\right|}{0.04}\right)^{2}\left(\frac{\left|W_{\tau e}^{e}\right|}{0.01}\right)^{2}\left(\frac{\tan \beta}{5.0}\right)^{2}
$$




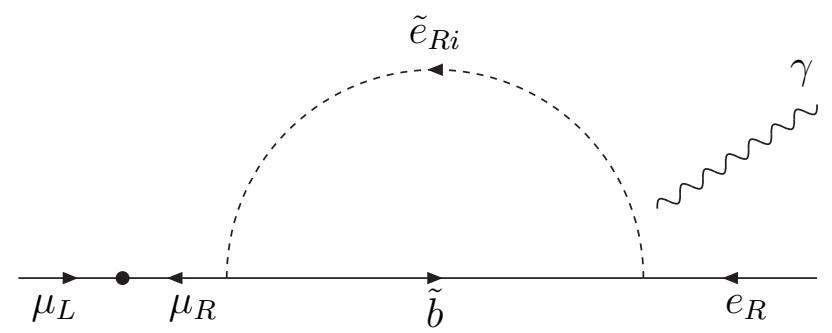

Figure 15: A Feynman diagram contributing to $\mu \rightarrow e \gamma$.

Here, we have normalized elements of the new mixing matrix $W^{e}$ by the corresponding values in the CKM matrix. This is well motivated because $W^{e}$ comes from a rotation of the right-handed charged leptons $e$, and the rotation of $e$ is expected to be similar to that of the left-handed quarks $q$, which determines the CKM matrix.

The prediction given in Eq. (14) is very interesting, since it gives a number close to the present experimental bound $\operatorname{Br}(\mu \rightarrow e \gamma) \lesssim 1.2 \times 10^{-11}$ [41]. While we expect an uncertainty of a factor of a few in the estimate of Eq. (14), we can still say that the present $\mu \rightarrow e \gamma$ decay experiment has already probed the theory up to about $\tilde{m} \simeq 200 \mathrm{GeV}(300 \mathrm{GeV})$ for $\tan \beta=5$ (10). Furthermore, a new experiment is under construction at PSI which aims for a sensitivity to $\operatorname{Br}(\mu \rightarrow e \gamma)$ at the $10^{-14}$ level [42]. From Eq. (14), $\operatorname{Br}(\mu \rightarrow e \gamma) \lesssim 10^{-14}$ corresponds to $\tilde{m} \gtrsim 1.5 \mathrm{TeV}(2 \mathrm{TeV})$ for $\tan \beta=5$ (10), so that this experiment will probe essentially all the parameter region of the theory where radiative electroweak symmetry breaking occurs naturally.

Another important lepton flavor violating process is $\tau \rightarrow \mu \gamma$ decay, which is predicted as

$$
\operatorname{Br}(\tau \rightarrow \mu \gamma) \simeq 5 \times 10^{-8}\left(\frac{200 \mathrm{GeV}}{\tilde{m}}\right)^{4}\left(\frac{\left|W_{\tau \mu}^{e}\right|}{0.04}\right)^{2}\left(\frac{\left|W_{\tau \tau}^{e}\right|}{1.0}\right)^{2}\left(\frac{\tan \beta}{5.0}\right)^{2}
$$

The present experimental bound comes from CLEO: $\operatorname{Br}(\tau \rightarrow \mu \gamma) \lesssim 1.1 \times 10^{-6}$ [43]. The $B$ factories at KEK and SLAC will improve the bound to the level of $10^{-7}$. Note that the combination of lepton flavor violation mixing angles, $\theta_{i j}^{e}$, appearing in Eq. (15) is different from that in Eq. (14). Therefore, in principle, we can determine all the lepton flavor violation mixing angles, $\theta_{12}^{e}$ and $\theta_{23}^{e}$, by measuring both $\mu \rightarrow e$ and $\tau \rightarrow \mu$ transition rates, if we know $\tilde{m}$ and $\tan \beta$ from independent measurements of the superparticle spectrum. These branching ratios, together with the branching ratio for $\mu \rightarrow$ eee and the rate for $\mu \rightarrow e$ conversion, are shown [3] in Table 1 for two representative values of $\tilde{m}$ and $\tan \beta$. 


\section{Conclusions}

We have proposed an alternative framework for physics at the scale of unification of the strong and electroweak interactions. The three pillars of physics beyond the standard model - grand unification, supersymmetry and extra dimensions - are combined in a way that allows calculations to a new level of precision. There are two keys to this framework: breaking of the unified gauge symmetry by local defects and the validity of the higher dimensional theory over a large energy range. The framework is illustrated in Figure 5. The geometrical breaking of gauge symmetry leads to a new constrained set of theories, and a high degree of calculability follows because the effective theory is valid up to the high energy scale of strong coupling and the spectrum of KK modes is determined.

Local gauge symmetry breaking defects could be viewed as a step backwards: the Higgs mechanism provides a dynamical origin for symmetry breaking resulting from an underlying theory that is completely symmetrical. Defects represent explicit local breaking of gauge symmetry. They arise from the assumed form for boundary conditions on the fields in extra dimensions, which are presumably to be determined by a more fundamental theory. However, in practice we find that the symmetry breaking boundary conditions provide a simple and elegant description of nature, making the Higgs fields and Higgs potentials of realistic 4D grand unified theories appear complicated and cumbersome.

The defect framework elegantly solves outstanding problems of $4 D$ unification, and the simplest model fits the data with extraordinary accuracy. The mass splitting of Higgs doublets and triplets is a necessary consequence of a bulk Higgs multiplet, proton decay from dimension five operators is forbidden by a spacetime symmetry of the bulk, and quark-lepton mass relations occur only for the heavy generation located on a symmetrical boundary.

The minimal model has an $S U(5)$ gauge symmetry in a $5 \mathrm{D}$ bulk, valid over the energy interval $M_{c} \simeq 5 \times 10^{14} \mathrm{GeV}$ to $M_{s} \simeq 1 \times 10^{17} \mathrm{GeV}$, as shown in Figure 10. The single 3-2-1 point defect leads to a revised picture of gauge coupling unification, illustrated in Figures 6 and 11. Since the physics between $M_{c}$ and $M_{s}$ is known, unified threshold corrections can be computed, yielding a successful prediction for the weak mixing angle of extraordinary precision: $\sin ^{2} \theta_{w}=0.2313 \pm 0.0004$. Proton decay by $X$ gauge boson exchange is governed by the scale $M_{c}$, and a lifetime of order $10^{34}$ years is expected for the mode $e^{+} \pi^{0}$. Predictions for other modes can also be made.

Further predictions depend on how supersymmetry is broken, and we have explored the consequences of having this breaking also follow from boundary defects in the same extra dimension. There are two supersymmetries in 5D, but the boundaries of the fifth dimension can support only a single supersymmetry. If there is a misalignment of the supersymmetries at the two boundaries by a small angle $\alpha$, as shown in Figures 13, supersymmetry is broken 
in the low energy $4 \mathrm{D}$ theory by an amount $\tilde{m}=\alpha M_{c}$. The entire superpartner spectrum can then be predicted in terms of $\tilde{m}$ and $\tan \beta$, as shown in Table 1. Furthermore, large tree-level lepton flavor violation is expected, leading to observable rates for $\mu \rightarrow e$ and $\tau \rightarrow \mu$ transitions. Finally, the supersymmetric threshold corrections to the quark-lepton mass relation $m_{b} / m_{\tau}$ can be computed. In the minimal $5 \mathrm{D}$ model this result is more successful than in the case of $4 \mathrm{D}$ unification, and is shown in Figure 14.

\section{Acknowledgments}

This work was supported in part by the Director, Office of Science, Office of High Energy and Nuclear Physics, of the U.S. Department of Energy under Contract DE-AC03-76SF00098, and in part by the National Science Foundation under grant PHY-00-98840. 


\section{References}

[1] L. J. Hall and Y. Nomura, Phys. Rev. D 64, 055003 (2001) [arXiv:hep-ph/0103125].

[2] L. J. Hall and Y. Nomura, Phys. Rev. D 65, 125012 (2002) [arXiv:hep-ph/0111068].

[3] L. J. Hall and Y. Nomura, Phys. Rev. D 66, 075004 (2002) [arXiv:hep-ph/0205067].

[4] H. Georgi and S. L. Glashow, Phys. Rev. Lett. 32, 438 (1974);

H. Georgi, H. R. Quinn and S. Weinberg, Phys. Rev. Lett. 33, 451 (1974).

[5] J. C. Pati and A. Salam, Phys. Rev. D 10, 275 (1974).

[6] H. Georgi, in Particles and Fields, edited by C.E. Carlson (AIP, New York, 1975), p. 575;

H. Fritzsch and P. Minkowski, Annals Phys. 93, 193 (1975).

[7] S. Dimopoulos and H. Georgi, Nucl. Phys. B 193, 150 (1981);

N. Sakai, Z. Phys. C 11, 153 (1981).

[8] S. Dimopoulos, S. Raby and F. Wilczek, Phys. Rev. D 24, 1681 (1981);

L. E. Ibanez and G. G. Ross, Phys. Lett. B 105, 439 (1981).

[9] N. Sakai and T. Yanagida, Nucl. Phys. B 197, 533 (1982);

S. Weinberg, Phys. Rev. D 26, 287 (1982).

[10] T. Goto and T. Nihei, Phys. Rev. D 59, 115009 (1999) [arXiv:hep-ph/9808255];

H. Murayama and A. Pierce, Phys. Rev. D 65, 055009 (2002) [arXiv:hep-ph/0108104].

[11] M. S. Chanowitz, J. R. Ellis and M. K. Gaillard, Nucl. Phys. B 128, 506 (1977);

A. J. Buras, J. R. Ellis, M. K. Gaillard and D. V. Nanopoulos, Nucl. Phys. B 135, 66 (1978).

[12] K. Hagiwara et al. [Particle Data Group Collaboration], Phys. Rev. D 66, 010001 (2002).

[13] P. Langacker and N. Polonsky, Phys. Rev. D 52, 3081 (1995) [arXiv:hep-ph/9503214];

J. Bagger, K. T. Matchev and D. Pierce, Phys. Lett. B 348, 443 (1995) [arXiv:hep$\mathrm{ph} / 9501277]$.

[14] L. J. Hall, H. Murayama and Y. Nomura, Nucl. Phys. B 645, 85 (2002) [arXiv:hepth/0107245].

[15] Y. Nomura, Phys. Rev. D 65, 085036 (2002) [arXiv:hep-ph/0108170].

[16] K. R. Dienes, E. Dudas and T. Gherghetta, Phys. Lett. B 436, 55 (1998) [arXiv:hepph/9803466]; Nucl. Phys. B 537, 47 (1999) [arXiv:hep-ph/9806292].

[17] Y. Nomura, D. R. Smith and N. Weiner, Nucl. Phys. B 613, 147 (2001) [arXiv:hep$\mathrm{ph} / 0104041]$. 
[18] L. J. Hall, Y. Nomura, T. Okui and D. R. Smith, Phys. Rev. D 65, 035008 (2002) [arXiv:hep-ph/0108071].

[19] T. Asaka, W. Buchmuller and L. Covi, Phys. Lett. B 523, 199 (2001) [arXiv:hep$\mathrm{ph} / 0108021]$.

[20] A. Hebecker and J. March-Russell, Nucl. Phys. B 625, 128 (2002) [arXiv:hep-ph/0107039].

[21] L. J. Hall and Y. Nomura, arXiv:hep-ph/0207079.

[22] P. Candelas, G. T. Horowitz, A. Strominger and E. Witten, Nucl. Phys. B 258, 46 (1985);

E. Witten, Nucl. Phys. B 258, 75 (1985);

J. D. Breit, B. A. Ovrut and G. C. Segre, Phys. Lett. B 158, 33 (1985).

[23] L. J. Dixon, J. A. Harvey, C. Vafa and E. Witten, Nucl. Phys. B 261, 678 (1985); Nucl. Phys. B 274, 285 (1986);

L. E. Ibanez, J. E. Kim, H. P. Nilles and F. Quevedo, Phys. Lett. B 191, 282 (1987).

[24] Y. Kawamura, Prog. Theor. Phys. 105, 999 (2001) [arXiv:hep-ph/0012125].

[25] A. Hebecker and J. March-Russell, Nucl. Phys. B 613, 3 (2001) [arXiv:hep-ph/0106166].

[26] L. J. Hall, Y. Nomura and D. R. Smith, Nucl. Phys. B 639, 307 (2002) [arXiv:hep$\mathrm{ph} / 0107331]$.

[27] L. Hall, J. March-Russell, T. Okui and D. R. Smith, arXiv:hep-ph/0108161.

[28] L. J. Hall, R. Rattazzi and U. Sarid, Phys. Rev. D 50, 7048 (1994) [arXiv:hep-ph/9306309];

R. Hempfling, Phys. Rev. D 49, 6168 (1994);

M. Carena, M. Olechowski, S. Pokorski and C. E. Wagner, Nucl. Phys. B 426, 269 (1994) [arXiv:hep-ph/9402253].

[29] T. Yanagida, in Proceedings of the Workshop on the Unified Theory and Baryon Number in the Universe, edited by O. Sawada and A. Sugamoto (KEK report 79-18, 1979), p. 95;

M. Gell-Mann, P. Ramond, and R. Slansky, in Supergravity, edited by P. van Nieuwenhuizen and D.Z. Freedman (North Holland, Amsterdam, 1979), p. 315.

[30] A. Hebecker and J. March-Russell, Phys. Lett. B 541, 338 (2002) [arXiv:hep-ph/0205143];

A. Hebecker, J. March-Russell and T. Yanagida, arXiv:hep-ph/0208249.

[31] N. Arkani-Hamed, A. G. Cohen and H. Georgi, Phys. Lett. B 516, 395 (2001) [arXiv:hepth/0103135].

[32] A. Hebecker and J. March-Russell, Phys. Lett. B 539, 119 (2002) [arXiv:hep-ph/0204037].

[33] L. J. Hall, V. A. Kostelecky and S. Raby, Nucl. Phys. B 267, 415 (1986).

[34] R. Barbieri, L. J. Hall and Y. Nomura, Phys. Rev. D 66, 045025 (2002) [arXiv:hep$\mathrm{ph} / 0106190]$. 
[35] R. Barbieri, L. J. Hall and Y. Nomura, Nucl. Phys. B 624, 63 (2002) [arXiv:hepth/0107004].

[36] D. Marti and A. Pomarol, Phys. Rev. D 64, 105025 (2001) [arXiv:hep-th/0106256];

D. E. Kaplan and N. Weiner, arXiv:hep-ph/0108001;

G. von Gersdorff and M. Quiros, Phys. Rev. D 65, 064016 (2002) [arXiv:hep-th/0110132].

[37] L. J. Hall, Y. Nomura and A. Pierce, Phys. Lett. B 538, 359 (2002) [arXiv:hep$\mathrm{ph} / 0204062]$.

[38] R. Contino, L. Pilo, R. Rattazzi and E. Trincherini, Nucl. Phys. B 622, 227 (2002) [arXiv:hep-ph/0108102].

[39] R. Barbieri and L. J. Hall, Phys. Lett. B 338, 212 (1994) [arXiv:hep-ph/9408406];

R. Barbieri, L. J. Hall and A. Strumia, Nucl. Phys. B 445, 219 (1995) [arXiv:hep$\mathrm{ph} / 9501334]$.

[40] J. Hisano, T. Moroi, K. Tobe and M. Yamaguchi, Phys. Lett. B 391, 341 (1997) [Erratumibid. B 397, 357 (1997)] [arXiv:hep-ph/9605296]; Phys. Rev. D 53, 2442 (1996) [arXiv:hep$\mathrm{ph} / 9510309]$.

[41] M. L. Brooks et al. [MEGA Collaboration], Phys. Rev. Lett. 83, 1521 (1999) [arXiv:hepex/9905013].

[42] L.M. Barkov et al., research proposal to PSI (1999);

S. Ritt, in Proceedings of the 2nd Workshop on Neutrino Oscillations and their Origin, edited by Y. Suzuki et al. (World Scientific, Singapore, 2001), p. 245.

[43] S. Ahmed et al. [CLEO Collaboration], Phys. Rev. D 61, 071101 (2000) [arXiv:hepex/9910060]. 